EDITORIALS

\title{
Will non-invasive markers replace liver biopsy for diagnosing and staging fibrosis in non-alcoholic steatohepatitis?
}

Etsuko Hashimoto* and Geoffrey C Farrell ${ }^{\dagger}$

* Department of Internal Medicine and Gastroenterology, Tokyo Women's Medical University, Tokyo, Japan; and ${ }^{\dagger}$ Gastroenterology and Hepatology Unit, ANU Medical School, Canberra, Australia

See article in J. Gastroenterol. Hepatol. 2009; 24: 564-568.

The recent dramatic increase of obesity and metabolic syndrome has caused a striking increase in non-alcoholic fatty liver disease (NAFLD) in both European and North American countries and Asia. ${ }^{1}$

Non-alcoholic fatty liver disease consists of two clinical entities: simple steatosis, which generally follows a benign nonprogressive clinical course; and non-alcoholic steatohepatitis (NASH), which may progress to cirrhosis and hepatocellular carcinoma. NASH was characterized and defined by Ludwig et al. in $1980 .^{2}$ The concept referred to subjects who did not consume alcohol but had progressive liver disease with liver biopsy findings similar to those of alcoholic hepatitis, and documented the clinical correlation with obesity and lifestyle-related diseases such as type 2 diabetes and hyperlipidemia. NASH emerged as a clinicopathological concept and even now biopsy evaluation is considered the gold standard for a definitive diagnosis.

The principal histological features of NASH are as follows: presence of macrovesicular steatosis, ballooning degeneration of hepatocytes, mixed lobular inflammation, Mallory hyaline, megamitochondria, acidophilic bodies, and glycogenated nuclei. Perivenular and perisinusoidal-pericellular fibrosis are characteristic features of NASH fibrosis. These features are predominant in zone 3 (perivenular areas) and develop from zone 3 to the panacinar zone. However, many pediatric cases have atypical features such as more zone 1 steatosis, little or no ballooning or Malory hyaline, and more portal-based chronic inflammation and fibrosis.

In 1999, Matteoni et al. found that cirrhosis and liver-related mortality were related to the histological characteristics of NAFLD. ${ }^{3}$ To evaluate the clinicopathological correlation, they divided 132 NAFLD patients into four categories based on the presence of specific histological lesions: type 1, fatty liver alone; type 2 , fatty liver plus lobular inflammation; type 3 , steatosis and ballooning degeneration; and type 4 , as in type 3 plus either Mallory hyaline or fibrosis. They confirmed the benign course of patients who had steatosis with or without lobular inflammation (types 1 and 2 NAFLD) and a progressive course for patients who had type 3 or 4 NAFLD. They defined type 3 and 4 as NASH and

Accepted for publication 12 January 2009

\section{Correspondence}

Professor Etsuko Hashimoto, 8-1 Kawada-cho, Shinjuku-ku, Tokyo 162-8666, Japan. Email: drs-hashimoto@mti.biglobe.ne.jp emphasized the importance of ballooning degeneration, which is a structural manifestation of microtubular disruption and severe cell injury. Matteoni's system served to distinguish simple steatosis (type 1 and 2) from NASH (type 3 and 4), but did not include an assessment of NASH severity.

In evaluating chronic hepatitis, necroinflammatory grade and fibrotic stage are distinguished. On this conceptual basis, Brunt et al. proposed a grading (steatosis and necroinflammatory changes) and staging (fibrosis) system for a semiquantitative evaluation of NASH in $1999 .{ }^{4}$ Unfortunately, this system applied only to NASH and it was not applicable to the entire spectrum of NAFLD.

In 2005, the NASH Clinical Research Network presented and validated a NAFLD activity score (NAS). ${ }^{5}$ This score was based on the classification proposed earlier by Brunt et al., it was applicable to adult and pediatric NAFLD patients and allowed the assessment of changes with therapy. The score is defined as the unweighted sum of the scores for steatosis (0-3), lobular inflammation (0-3) and ballooning (0-2); thus, NAS ranges from $0-8$. A score of more than 5 correlated with a diagnosis of NASH, and biopsies with scores of less than 3 were diagnosed as 'not NASH'. Patients who had scores of 3 or 4 were borderline. Fibrosis was not included in NAS to maintain the paradigm of distinguishing grade from stage. Regarding stage, stage 1 was extended to include a distinction between delicate (1A) and dense (1B) perisinusoidal fibrosis in zone 3 , and to detect portal-only fibrosis, without perisinusoidal fibrosis (stage 1C). Stage 2 was characterized by additional evidence of focal or extensive periportal fibrosis. Stage 3 was characterized by bridging fibrosis, and stage 4 was cirrhosis. Because NAS and staging allow the diagnosis of the entire range of NAFLD, it may prove useful in standardizing the histological diagnosis of NAFLD in clinical trials.

A liver biopsy remains the only reliable means to precisely diagnose NASH and establish the severity of liver injury and presence of fibrosis. However, liver biopsy has several drawbacks: it is an invasive, painful and costly procedure, with the possibility of sampling error and variability in pathologist interpretation. Moreover, given the extremely high prevalence of NAFLD in the general population, a liver biopsy is poorly suited as a diagnostic test for NAFLD. These shortcomings and drawbacks of liver biopsy, as recognized by a recent Asia-Pacific Consensus on NAFLD,${ }^{6}$ support the urgent need to find non-invasive markers for the assessment of NASH.

An ideal biomarker should be simple, accurate, reproducible, inexpensive and readily available. Currently, the NAFLD biomarkers have been evaluated for: (i) distinguishing NASH from NAFLD; and/or (ii) diagnosing advanced fibrosis or cirrhosis (Fig. 1). The study subjects have varied from the general population to NASH patients assessed in liver clinics.

Serum transaminases are usually mildly elevated in NAFLD, however, their utility for the diagnosis of NASH is poor. The process of development of NASH has been referred to as the two-hit hypothesis, with steatosis being the first hit and subsequent hepatocellular injury causing NASH the second hit. Insulin resistance, excess intracellular fatty acids, imbalance of cytokine production, oxidative stress, lipid peroxidation, iron overload, depletion of adenosine triphosphate and mitochondrial dysfunction are candidate pathways for induction of hepatocellular injury (NASH) in a steatotic liver. Accordingly, many studies have been focused on the second hit in NAFLD; namely, thioredoxin as a marker of oxidative stress, cytokines (tumor necrosis factor- $\alpha^{7}$ and interleukin-6), adiponectin, leptin and C-reactive protein, among 


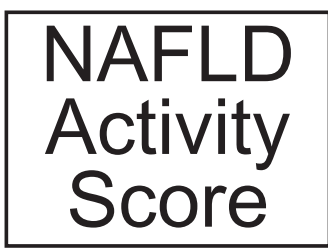

\section{Fibrosis Stage}

(steatosis, ballooning, inflammation)

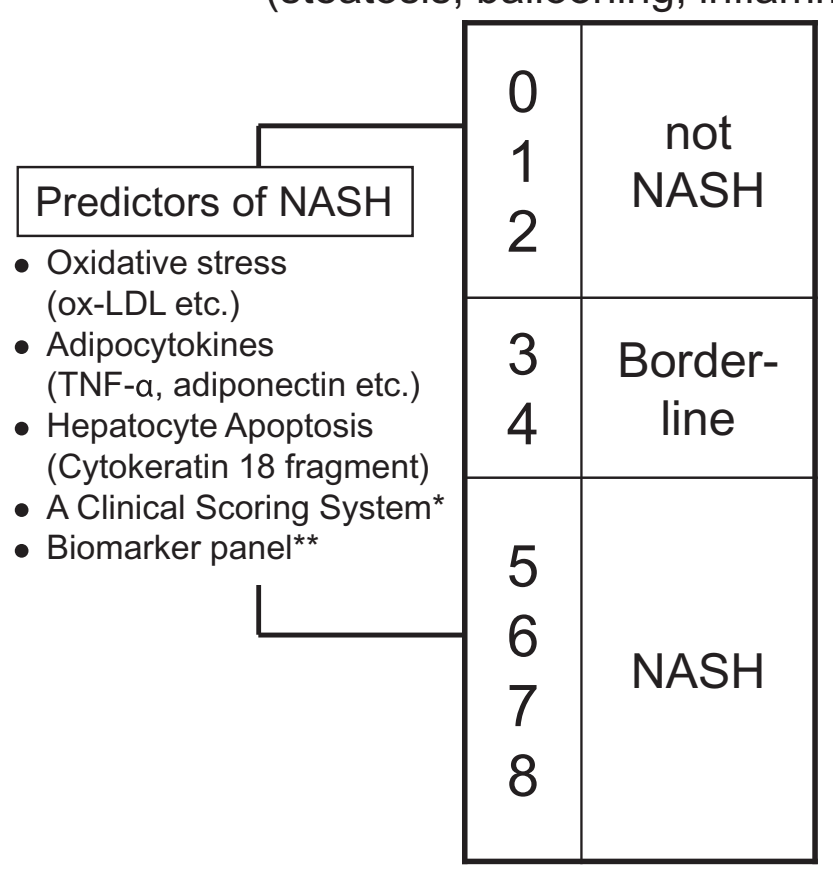

*; hypertension, type 2 diabetes, sleep apnea, AST, ALT, non-Black (ref.12). **; adiponectin, resistin, cytokeratin 18 fragments (ref.13).

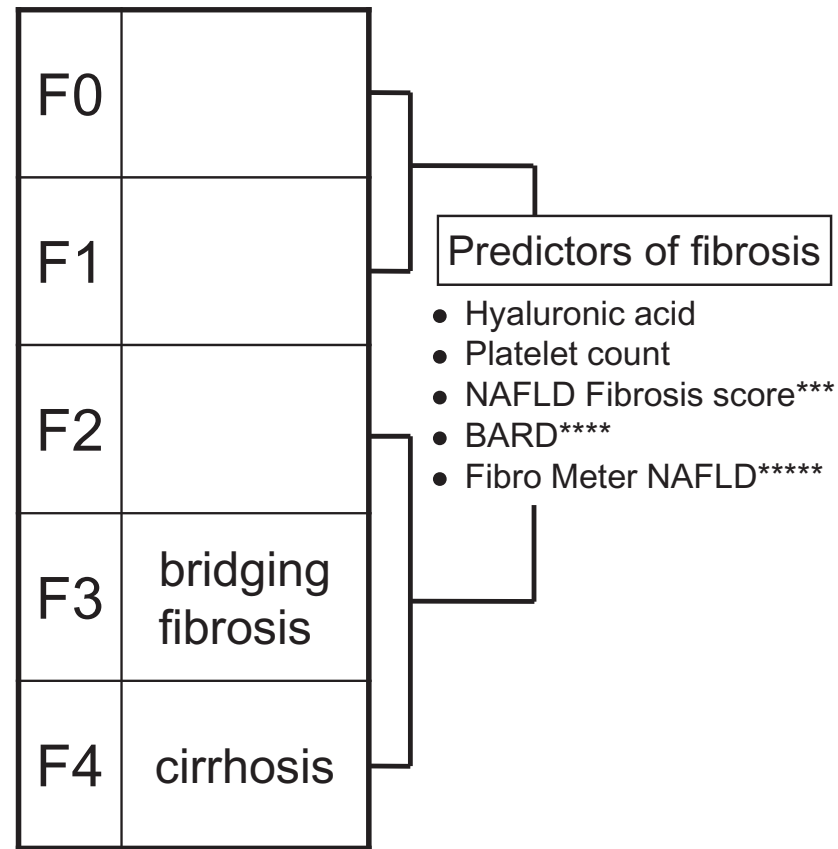

***; age, hyperglycemia, BMI, platelet count, albumin, AST/ALT (ref.15). ****; BMI, AST/ALT, type 2 diabetes (ref.16).

*****; glucose, AST, ferritin, platelet count, ALT body weight, age (ref.17).

Figure 1 Serum and clinical predictors of NASH and/or fibrosis in NAFLD. ALT, alanine aminotransferase; AST, aspartate aminotransferase; BMI, body mass index; LDL, low-density lipoprotein; NAFLD, non-alcoholic fatty liver disease; NASH, non-alcoholic steatohepatitis; TNF, tumor necrosis factor.

others. The relationship between these markers and NASH is inconsistent, because they were found to correlate more closely with the degree of steatosis or necroinflammatory activity than with the fibrotic stage.

Hepatocyte apoptosis has been found to be a prominent feature of NASH. Wieckowska et al. reported that serum caspase 3-generated cytokeratin-18 (CK-18) fragment levels, a product of hepatocyte apoptosis in the injured liver, independently predicted the presence of NASH and correlated with the magnitude of hepatocyte apoptosis and disease severity. ${ }^{8}$ This study was validated in a large number of NAFLD patients by the NASH Clinical Research Network. ${ }^{9}$ Diab et al. also assessed the usefulness of plasma CK-18 fragments in a cohort of patients coming to bariatric surgery. ${ }^{10}$ Their results support the potential utility of this test for diagnosis and staging of NAFLD. Moreover, they showed a significant decrease in CK-18 fragment levels in most patients 6 months after bariatric surgery.

The work by Malik and coworkers in this issue of the Journal reconfirms the usefulness of CK-18 fragments. ${ }^{11}$ They evaluated the predictive value of serum markers to identify NASH and liver fibrosis. They evaluated CK-18 fragments, a hyaluronic acid tissue inhibitor of metalloproteinase 1, and YKL-40. They found that only CK-18 fragments could identify NASH. Accordingly, the CK-18 fragments are expected to become an ideal biomarker for $\mathrm{NASH}$, although currently it is not routinely available as a laboratory test.

To increase the accuracy of non-invasive markers to diagnose $\mathrm{NASH}$, multiple serum markers have been combined into mathematical models to obtain predictive scores. In 2008, two clinical scoring systems for predicting NASH in patients with morbid obesity have been reported. Campos et al. identified hypertension, type 2 diabetes, the presence of sleep apnea, elevated transaminases and non-black race as independent predictors of NASH. ${ }^{12}$ The other one was described by Younossi et al. ${ }^{13}$ It was based on serum levels of adiponectin, resistin and CK-18 fragments.

Concerning fibrosis, several routinely available laboratory tests serve to determine the cirrhotic stage. We have reported that elevation of serum hyaluronic acid levels is seen in stage 3 and 4 NAFLD patients, and platelet count decreased in stage 4 NAFLD patients; these findings indicate that hyaluronic acid can predict stage fibrosis 
at an earlier stage than platelet count. ${ }^{14}$ Extracellular matrix proteins, such as hyaluronic acid and collagen components, have been examined as potential markers of liver fibrosis in NAFLD. Several models to predict the extent of fibrosis in NAFLD patients have been reported. ${ }^{15-17}$ In general, most of these show similar accuracy for the detection of advanced fibrosis, but weak accuracy for that of mild fibrosis. This represents a limitation for the screening of patients with NAFLD. On the other hand, NAFLD patients with mild fibrosis might benefit the most from therapeutic interventions. Several imaging techniques have also been advocated as noninvasive diagnostic tests for NASH. In addition, ultrasonographybased transient elastography or FibroScan, have shown promising results regarding the severity of liver fibrosis.

In summary, liver biopsy currently remains the gold standard for the diagnosis of NASH, including assessment of the fibrotic severity of this common liver disease. In the future, improved understanding of the pathogenesis of NASH and new technologies, such as CK-18 fragments and serum hyaluronic acid, and FibroScan should contribute to the diagnosis of NASH and hopefully become a reliable non-invasive alternative to liver biopsy.

\section{References}

1 Amarapurkar DN, Hashimoto E, Lesmana LA et al. How common is non-alcoholic fatty liver disease in the Asia-Pacific region and are there local differences? J. Gastroenterol. Hepatol. 2007; 22: 788-93.

2 Ludwig J, Viggiano TR, McGill DB et al. Nonalcoholic steatohepatitis. Mayo Clinic experiences with a hitherto unnamed disease. Mayo Clin. Proc. 1980; 55: 434-8.

3 Matteoni CA, Younossi ZM, Gramlich T et al. Nonalcoholic fatty liver disease: a spectrum of clinical and pathological severity. Gastroenterology 1999; 116: 1413-19.

4 Brunt EM, Janney CG, DiBisceglie AM et al. Nonalcoholic steatohepatitis: a proposal for grading and staging the histological lesions. Am. J. Gastroenterol. 1999; 94: 2467-74.

5 Kleiner DE, Brunt EM, Van Natta M et al. Nonalcoholic Steatohepatitis Clinical Research Network. Related Articles: design and validation of a histological scoring system for nonalcoholic fatty liver disease. Hepatology 2005; 41: 1313-21.

6 Farrell GC, Chitturi S, Lau GKK et al. Guidelines for the assessment and management of non-alcoholic fatty liver disease in the Asia-Pacific region: executive summary. J. Gastroenterol. Hepatol. 2007; 22: 775-7.

7 Tokushige K, Takakura M, Hashimoto E et al. Influence of TNF gene polymorphisms in Japanese patients with NASH and simple steatosis. J. Hepatol. 2007; 46: 1104-10.

8 Wieckowska A, Zein N, Yerian LM et al. In vivo assessment of liver cell apoptosis as a novel biomarker of disease severity in nonalcoholic fatty liver disease. Hepatology 2006; 44: 27-33.

9 Wieckowska A, Lopez AR, Zein NN et al. Noninvasive assessment of hepatocyte apoptosis in nonalcoholic fatty liver disease; a multi-center validation study. Gastroenterology 2007; 132: A729.

10 Diab D, Yerian L, Schauer P et al. Cytokeratin 18 fragment levels as a noninvasive biomarker for nonalcoholic steatohepatitis in bariatric surgery patients. Clin. Gastroenterol. Hepatol. 2008; 6: 1249-54.

11 Malik R, Chang M, Bhaskar K et al. The clinical utility of biomarkers and the nonalcoholic steatohepatitis CRN liver biopsy scoring system in patients with nonalcoholic fatty liver disease. $J$. Gastroenterol. Hepatol. 2009; 24: 564-8.

12 Campos GM, Bambha K, Vittinghoff E et al. A clinical scoring system for predicting nonalcoholic steatohepatitis in morbidly obese patients. Hepatology 2008; 47: 1916-23.
13 Younossi ZM, Jarrar M, Nugent C et al. A novel diagnostic biomarker panel for obesity-related nonalcoholic steatohepatitis (NASH). Obes. Surg. 2008; 18: 1430-7.

14 Kaneda H, Hashimoto E, Yatsuji S et al. Hyaluronic acid levels can predict severe fibrosis and platelet counts can predict cirrhosis in patients with nonalcoholic fatty liver disease. J. Gastroenterol. Hepatol. 2006; 21: 1459-65.

15 Angulo P, Hui JM, Marchesini G et al. The NAFLD fibrosis score: a noninvasive system that identifies liver fibrosis in patients with NAFLD. Hepatology 2007; 45: 846-54.

16 Harrison S, Oliver D, Arnold $\mathrm{H}$ et al. Development and validation of a simple NAFLD clinical scoring system for identifying patients without advanced disease. Gut 2008; 57: 1441-7.

17 Cales P, Laine F, Boursier J et al. Comparison of blood tests for liver fibrosis specific or not to NAFLD. J. Hepatol. 2009; 50: 165-73.

\section{Colorectal cancer in inflammatory bowel disease}

\author{
Rupert W L Leong and Jenn H Koo
}

\begin{abstract}
Gastroenterology and Liver Services, Bankstown-Lidcombe and Concord Hospitals, Sydney South West Area Health Service, Sydney, Australia
\end{abstract}

See article in J. Gastroenterol. Hepatol. 2009; 24: 667-671.

Colorectal (CRC) cancer is one of the most devastating complications of chronic colitis in the setting of inflammatory bowel disease (IBD). Only 1-2\% of CRC occur in IBD patients but IBD is one of the major risk factors of CRC, and up to $15 \%$ of IBD mortality has been attributed to CRC. ${ }^{1}$

Current strategies in the reduction or management of colitisassociated CRC include chemoprophylaxis, colonoscopy surveillance of at risk individuals and proctocolectomy is a potentially curative treatment for those with precancerous dysplasia or early cancer. Colonoscopy surveillance is recommended after 8 to 10 years of extensive colitis and 12 to 15 years of left-sided colitis. ${ }^{2}$ The detection of colorectal dysplasia is considered a strong predictor of CRC in IBD, and there may be some emerging evidence of improved survival in those who underwent surveillance. ${ }^{3}$ The yield of detecting dysplasia is variable. Friedman et al. found a $25 \%$ rate of definite dysplasia after the tenth examination when surveillance is performed every 1-2 years. ${ }^{4}$ Despite adherence to surveillance programs, CRC may still develop. Lutgens et al. showed that $17-28 \%$ of CRC are missed despite adherence to the British Society of Gastroenterology and American Gastroenterology Association guidelines, mostly due to CRC occurring prior to guideline-based surveillance starting points. ${ }^{5}$ A high proportion of cancers are also found as interval cancers outside of surveillance programs, raising the possibility of missed early lesions during

Accepted for publication 12 January 2009.

\section{Correspondence}

Associate Professor Rupert WL Leong, Director of Endoscopy, Ambulatory Care Endoscopy Unit, Level 1 West, Hospital Rd, Concord, NSW 2139, Australia. Email: rupertleong@hotmail.com 